Uncoupling proteins 2 and 3 are fundamental for mitochondrial $\mathrm{Ca}^{2+}$ uniport.

Trenker, M. et al. Nature Cell Biol. 9, 445-452 (2007)

Under physiological conditions, mitochondria take up $\mathrm{Ca}^{2+}$ from the cytosol; however, the mechanism of mitochondrial import is unknown. Trenker et al. show that uncoupling protein-2 (UCP2) and UCP3 are essential for mitochondrial $\mathrm{Ca}^{2+}$ import. Overexpression of UCP2 or UCP3 enhanced mitochondrial $\mathrm{Ca}^{2+}$ uptake. $\mathrm{Ca}^{2+}$ uptake was inhibited by expression of dominantnegative mutants of UCP2 or UCP3, by depletion of UCP2 or UCP3 (mediated by small interfering RNAs) or in isolated Ucp $2^{-/-}$ mitochondria. By contrast, modulation of UCP1 expression had no effect on $\mathrm{Ca}^{2+}$ dynamics. It is unclear whether UCP2 and UCP3 form a channel in the inner mitochondrial membrane or whether they function indirectly to regulate the passage of $\mathrm{Ca}^{2+}$ ions.

\title{
DNA REPAIR
}

The structural determinants of checkpoint activation. MacDougall, C. A. et al. Genes Dev. 21, 898-903 (2007)

The ATR (ATM and Rad3-mediated) kinase is a central sensor of many forms of genotoxic stress that activates a DNA-damage checkpoint through its downstream effector checkpoint kinase-1 (Chk1). Using Xenopus laevis egg extract, MacDougall et al. identified a minimal DNA structure that activated the ATR-mediated checkpoint response. The authors found that single-stranded (ss) DNA with short complementary DNA sequences annealed along it was sufficient for checkpoint activation by ATR. This primed ssDNA structure is similar to those that are often formed following many types of DNA damage. Importantly, the authors have generated a tool that can be used to probe ATR-dependent checkpoint signalling; for example, the length of ssDNA next to the primer influences the extent of Chk1 activation. In addition, this forms a starting molecule from which the structural determinants that are required for checkpoint activation can be refined.

\section{$\Rightarrow$ OXYGEN SENSING}

HIF-1 regulates cytochrome oxidase subunits to optimize efficiency of respiration in hypoxic cells.

Fukuda, R. et al. Cell 129, 111-122 (2007)

Hypoxia-inducing factor-1 (HIF1) is activated under low-oxygen conditions to promote pathways of anaerobic metabolism. The authors show that, in mammalian cells, HIF1 also modulates aerobic metabolism by regulating the production of a catalytic subunit in the mitochondrial electron-transport chain - cytochrome c oxidase-4 (COX4). Under normal conditions, cells predominantly produce COX4-1, an isoform that generates moderate amounts of harmful reactive oxygen species (ROS). Under hypoxic conditions, HIF1 induces the production of COX4-2 and activates a mitochondrial LON protease that degrades COX4-1. COX4-2 is a more efficient isoform that enhances aerobic respiration during periods of hypoxic stress and generates lower levels of ROS. However, forced overproduction of COX4-2 under non-hypoxic conditions was more harmful to cells than was forced overproduction of COX4-1, which may explain why COX4-2 production is usually kept to low levels. The swapping of cytochrome oxidase subunits also occurs in yeast. Yeast lack a HIF1 homologue and regulate subunit production by a different mechanism, which provides a clear example of convergent evolution in eukaryotes. 